\title{
Electrothermal Bipolar Vessel Sealing System Dissection Reduces Drainage Fluid Output or Time to Drain Removal Following Axillary and Ilio-Inguinal Node Dissection in Melanoma Patients: A Pilot Study
}

\author{
Di Monta Gianluca, Marone Ugo*, Alfonso Amore, Benedetto Lucia, Caracò Corrado \\ Unit of Surgery “Melanoma-Skin Tumors”, Istituto Nazionale per lo Studio e la Cura dei Tumori “Fondazione G. Pascale”, Via \\ Mariano Semmola, Naples, Italy \\ Email: *u.marone@istitutotumori.na.it
}

How to cite this paper: Gianluca, D.M., Ugo, M., Amore, A., Lucia, B. and Corrado, C. (2018) Electrothermal Bipolar Vessel Sealing System Dissection Reduces Drainage Fluid Output or Time to Drain Removal Following Axillary and Ilio-Inguinal Node Dissection in Melanoma Patients: A Pilot Study. Journal of Cancer Therapy, 9 , 613-622.

https://doi.org/10.4236/jct.2018.99053

Received: April 13, 2018

Accepted: September 2, 2018

Published: September 5, 2018

Copyright $\odot 2018$ by authors and Scientific Research Publishing Inc. This work is licensed under the Creative Commons Attribution International License (CC BY 4.0).

http://creativecommons.org/licenses/by/4.0/

(c) (i) Open Access

\begin{abstract}
Background: Radical lymph node dissection (RLND) is the treatment of choice in stage III melanoma patients. Over half of the observed complications were related to post-operative serum collection affecting wound healing. The aim of this study was to evaluate the use of the LigaSure ${ }^{\mathrm{TM}}$ Small Jaw (LSJ) on postoperative drainage fluid volume and drainage permanence in patients undergoing RLND. Study design: This was a prospective single-center study in which consecutive melanoma patients underwent nodal dissection using the LSJ. Daily drainage volume and duration of drainage were recorded in post-operative course on days 3,10 or 15 and day 20. Results: A total of 70 patients were included. Mean postoperative drainage volume was lower. Around two-thirds of patients had a drainage fluid volume of less than $50 \mathrm{cc}$ at 10 days. Mean time to drainage removal was 14.5 days. Conclusions: This is the first report of nodal dissection in patients with cutaneous melanoma using an electrothermal bipolar vessel sealing system. The technique was feasible, safe, and effective and resulted in reduced drainage fluid volume and drainage permanence.
\end{abstract}

\section{Keywords}

Lymph Node Metastases, Lymphatic Drainage, Seroma Formation, Cutaneous Melanoma 


\section{Introduction}

Radical lymph node dissection (RLND) is the treatment of choice for patients with stage III melanoma. However, the incidence of complications after ilio-inguinal and axillary lymphadenectomy can be high, with local complication rates of up to $80 \%$ after ilio-inguinal RLND and up to $48 \%$ after axillary RLND [1] [2]. Over half of these complications are typically related to delayed wound healing as a result of postoperative seroma formation [3] [4]. Intraoperative insertion of closed suction drains is the traditional method used to reduce seroma formation and subsequent wound healing complications after any nodal dissection. However, their use can be associated with discomfort, increased risk of infection and restricted mobility, thereby delaying patients' return to normal activities. As such, other strategies to prevent seroma formation may be beneficial in improving postoperative recovery and quality of life of patients.

Various methods to reduce seroma formation have previously been proposed, all of which aim to reduce dead space and seal leaking capillaries and lymphatic channels. These approaches include tacking sutures [5], delayed exercise [6] [7], tetracycline spray [8], ultrasound cutting devices [9], bovine thrombin [10] and fibrin sealants [11] [12]. LigaSure ${ }^{\mathrm{TM}}$ vessel-sealing system (Covidien, USA) is a bipolar electrosurgical device that provides hemostasis by denaturing collagen and elastin from the vessel wall and surrounding connective tissue. The LigaSure Small $\mathrm{Jaw}^{\mathrm{TM}}$ instrument is designed to be used in confined surgical spaces, especially in thyroid surgery, parotid gland surgery and head and neck surgery, to usefully seal and cut vessels up to $7 \mathrm{~mm}$ in diameter. The efficacy and safety of the LigaSure ${ }^{\mathrm{TM}}$ electrothermal bipolar vessel sealing system is approved worldwide for supportive treatment in surgery to improve hemostasis [13]. However, it is not approved for the reduction of seroma output in patients undergoing nodal dissection.

In the present study, we analyzed our experience at the National Cancer Institute of Naples using the LigaSure Small Jaw (LSJ) vessel sealing system in patients with lymph nodal metastases from cutaneous melanoma undergoing axillary or ilio-inguinal dissection. The aim of the study was to evaluate whether this technique could decrease postoperative drainage fluid volume and time to drainage removal. These data represent the first report of the use of electrothermal bipolar vessel sealing in patients with melanoma undergoing nodal dissection.

\section{Material and Methods}

This was a prospective study carried out at a single center (Unit of Surgery "Melanoma-Skin Tumors", Istituto Nazionale per lo Studio e la Cura dei Tumori "Fondazione G. Pascale", Naples, Italy) which included consecutive patients with stage III melanoma who underwent radical axillary or ilio-inguinal dissection using the LSJ vessel sealing instrument (Covidien, USA) between June 2014 and July 2015. The study was conducted in accordance with the Declaration of Helsinki and Good Clinical Practice. All patients provided written informed consent.

Patients were eligible for the study if they were candidates for surgical treat- 
ment of metastatic stage III melanoma by axillary or ilio-inguinal RLND after positive sentinel node biopsy or clinically palpable disease.

Surgical technique: Surgical procedure was the same in patients after positive sentinel node biopsy or clinically palpable disease with the use of the LSJ electrothermal bipolar vessel sealing system instead of standard electrocautery to dissect fatty tissue, aponeurosis and to prepare the vascular plane.

Axillary dissection was carried out through a transverse/S-shaped incision, raising $1 \mathrm{~cm}$ thick skin flaps using the LSJ instrument to the lateral border of the pectoralis major and latissimus dorsi muscles. RLND was realized on level I, II and III, en bloc with supra-axillary fat pad, preserving the thoracodorsal neurovascular bundle and the long thoracic nerve. Major vessels, but not lymphatic vessels, were ligated, while fat dissection was carried out with the LSJ. At the end of surgery, a closed suction drain was inserted through the inferior skin flap and into the axilla.

Ilio-inguinal RLND was initiated with a lazy $S$ incision extending from the anterior superior iliac spine to the apex of the femoral triangle. Skin flaps were raised using the LSJ medially to the pubic tubercle and to the adductor longus muscle, and laterally to the apex of the femoral triangle. The fatty, node-bearing tissue of the triangle was removed, skeletonizing the femoral bundle in a subadventitial plane and transecting the saphenous vein at the confluence. Access to the iliac fossa was obtained via a transverse incision through the external oblique aponeurosis, $4 \mathrm{~cm}$ above the inguinal ligament, lifting up the peritoneum. RLND was performed around the external iliac vessels up to the level of the ureter and down in the pelvis to the obturator nerve. Finally, the muscular and aponeurosis edges were sutured, and the wound closed over a closed suction drain in the femoral triangle.

Postoperative care: All patients were instructed to reduce physical activity for the first week, to shower regularly, to record daily drainage fluid volume and to return to the hospital for weekly follow-up visits with a dedicated outpatient nurse team. Drainage was removed when 24-hour drainage fluid volume was less than $50 \mathrm{cc}$ for two consecutive days (with patients instructed to return to the hospital the day after the first day drainage volume was $<50 \mathrm{cc}$ ) or a maximum of 30 days after surgery.

Statistical analyses: The primary endpoint of the study was to evaluate the impact of the LSJ technique on postoperative drainage fluid output, with daily drainage volume measured on postoperative days 3, 10, 15 and 20. The secondary endpoint was time to drainage removal. Both endpoints were compared with the historical control group of our previous study [14] using descriptive statistics, in which 33 patients of the control group received standard surgical treatment with conventional electrocautery only (axillary metastases, $n=21$; inguinal metastases, $n=12$ ). The two treatment groups were comparable with regard to age, sex, number of removed lymph nodes and number of positive lymph nodes (Table 1) Primary baseline characteristics, number of lymph nodes removed, 
and numbers of positive lymph nodes were recorded for all patients.

\section{Results}

A total of 70 consecutive patients (Table 1) with melanoma underwent axillary or ilio-inguinal RLND, of whom 39 had axillary metastases and 31 had inguinal metastases. The median age of included patients was 59 years (range 29 - 82). Median number of lymph nodes removed was $22(15-29)$ and median number of positive lymph nodes was 2 (1 - 25).

Postoperative lymph output. Mean daily drainage on postoperative days 3, 10,15 and 20 was $112 c c, 53 c c, 25 c c$ and $17 c c$ respectively (Table 2). About $66.6 \%$ of patients had less than $55 \mathrm{cc}$ daily drainage volume at postoperative day 10.

Drainage removal incidence: Mean duration of closed suction drain placement was 14.5 days. A total of $32.4 \%$ of patients had drainage removed within 10 days and $67.5 \%$ of patients had drainage removed within 15 days (Table 3 ).

Table 1. Patient characteristics.

\begin{tabular}{|c|c|c|}
\hline & LSJ group $n=70$ & Standard treatment $(\mathrm{n}=33)$ \\
\hline Gender female/male (n) & $47 / 23$ & $12 / 21$ \\
\hline Age, median (years) & 59 & 64 \\
\hline mean & 44 & \\
\hline range & $29-82$ & 26,84 \\
\hline \multicolumn{3}{|l|}{ Breslow depth, (mm) } \\
\hline Median & 3.0 & \\
\hline Mean & 3.56 & \\
\hline Range & $0.6-10.0$ & \\
\hline \multicolumn{3}{|l|}{ Ulceration } \\
\hline Yes & 29 & \\
\hline No & 41 & \\
\hline \multicolumn{3}{|l|}{ Type of metastatic disease } \\
\hline Positive SN & 36 & 8 \\
\hline Clinical disease & 34 & 25 \\
\hline \multicolumn{3}{|l|}{ Site of dissection } \\
\hline Axilla & 39 & 21 \\
\hline Groin & 31 & 12 \\
\hline \multicolumn{3}{|l|}{ Nodal removed (n) } \\
\hline Mean & 22 & 19 \\
\hline Range & $15-29$ & $10-34$ \\
\hline \multicolumn{3}{|l|}{$\mathrm{N}^{\circ}$ positive node removed $(\mathrm{n})$} \\
\hline Mean & 2 & 1 \\
\hline Range & $1-25$ & $0-15$ \\
\hline
\end{tabular}


Table 2. Postoperative lymph output in patients treated with electrothermal bipolar vessel sealing system on postoperative days $3,10,15$ and 20 .

\begin{tabular}{cc}
\hline \multirow{2}{*}{ Postoperative day } & \multicolumn{2}{c}{ Mean drainage volume $(\mathrm{cc})$} \\
\cline { 2 - 2 } & Liga Sure vessel sealing system $(\mathrm{n}=70)$ \\
\hline Day 3 & 112 \\
Day 10 & 53 \\
Day 15 & 25 \\
Day 20 & 17 \\
\hline
\end{tabular}

Table 3. Duration of closed suction drain placement in patients treated with electrothermal bipolar vessel sealing system.

\begin{tabular}{ccc}
\hline & $\begin{array}{c}\text { LigaSure vessel } \\
\text { sealing system (n=70) }\end{array}$ & $\begin{array}{c}\text { Standard } \\
\text { treatment }(\mathrm{n}=33)\end{array}$ \\
\hline Mean number of days to drainage removal & 14.5 & 23 \\
Drainage-free patients at 10 Days (\%) & 32.4 & $3 \%$ \\
Drainage-free patients at 15 Days (\%) & 67.5 & $9 \%$ \\
\hline
\end{tabular}

There were no intraoperative complications or blood loss in either group. Two patients in the standard treatment control group had a dehiscence after ileo-inguinal dissection; one because of partial skin flap necrosis and one because of wound infection. No other major postoperative complications occurred and there was no perioperative mortality.

\section{Discussion}

Nodal status is the strongest single prognostic factor in patients with cutaneous melanoma and the mainstay of treatment is surgical RLND of the involved basin, after either positive sentinel node biopsy or detection of clinically palpable disease. Despite the treated basin, the procedure is considered high-risk in terms of postoperative wound complications, which can include seroma, infection, skin-edge necrosis and wound breakdown and which have been reported to occur in $25 \%-80 \%$ of patients [1] [2].

Fluid collection in the wound is one of the most common complications after RLND. The pathophysiology of seroma formation is not clearly understood and different hypotheses have been proposed, largely based on evidence from patients undergoing axillary dissection after mastectomy. Woodworth et al. suggested that seroma formation is a consequence of surgical disruption of lymphatics and capillaries with ensuing leakage of fluid into the dead space created by surgical dissection. In others studies, seroma is considered to be a consequence of inflammatory exudates, showing high concentrations of proteins in fluid aspirates [15]. Previous studies suggest that the bulk of the fluid accumulation after RLND is not simply lymph from divided lymphatic vessels. However, neither it is merely a collection of serum, but rather consists of fluid formed by 
acute inflammatory exudates in response to surgical trauma and the acute phase of wound healing [16] [17].

Following RLND, the use of closed suction drainage is required to evacuate lymph produced in the operative field. The duration of drainage in patients with melanoma can differ widely but is generally between 4 to 6 weeks [2] [18]. The use of electrocautery has been reported to be associated with a $44 \%$ increase in the probability of complications (including seroma, wound infection, wound necrosis, hematoma, and lymphedema) when compared with cold-knife technique [19]. Postoperative wound drainage has a significant negative impact on patients' well-being, interfering with their normal daily activities, reducing sleep and delaying their return to work. In a study of melanoma patients who had undergone inguino-femoral lymph node dissection, Mortenson and coworkers found that getting dressed, bathing, and sleeping were major problems for $67 \%$, $78 \%$ and $72 \%$ of patients, respectively [2].

Different surgical techniques, including avoidance of electrocautery, tacking sutures, ultrasound cutting devices, and modified lines of incision, have proven largely ineffective in preventing or reducing fluid collection after RLND [5] [9]. The addition of liquid fibrin sealants has also been disappointing in lymphadenectomy for melanoma [2] [18] [20]. In a previous randomized trial, the use of a fibrinogen/thrombin-coated collagen sealant patch reduced time to drainage removal by 4 days versus electrocautery ( 20 versus 23.3 days; $\mathrm{p}=0.002$ ) although daily drainage volume was not significantly reduced [14].

Energy-based ligation and cutting tools such as electrothermal bipolar vessel sealing systems are commonly used in minimally invasive or open surgical procedures. The advantages of these tools include permanent sealing, reduced foreign body inflammatory reaction, minimal thermal effect on surrounding tissues, and reduced tissue tangling and carbonization. They also appear to be effective in closing lymphatic vessels that normally remain open with electrocautery. These energy-based devices allow effective reduction of surgery time and safe tissue removal with no increase in postsurgical complications. The LSJ electrothermal bipolar vessel sealing system uses electrothermal energy to denature collagen and elastin within the vessel wall and surrounding connective tissue and is reported to take 3.5 seconds to seal a $7-\mathrm{mm}$ vessel in diameter with a blade temperature that never exceeds $80^{\circ} \mathrm{C}$.

Since the introduction of the LSJ instrument in 2010, many studies have compared it with conventional surgical methods especially as a hemostatic device in general surgery, urology, gynecology, thyroidectomy and axillary dissection [21]. In a meta-analysis of prospective randomized trials that compared the LigaSure electrothermal bipolar vessel sealing system with either clamping with suture ligation/electrocauterization or the harmonic scalpel in various surgical procedures, operative time was reduced and its use was associated with less blood loss, fewer complications, and reductions in postoperative pain [22]. In a prospective, randomized clinical study, the use of the LigaSure vessel sealing system for axillary dissection in breast cancer surgery was associated with signif- 
icantly reduced intraoperative blood loss, duration of axillary surgery, days of suction drainage, and length of hospital stay when compared with conventional axillary dissection using electrocautery, suture ligation or vascular clips [23]. In another recent analysis, the use of LigaSure was shown to be effective in reducing drain volume and duration of drain placement compared with conventional electrocautery in patients with breast cancer after axillary dissection [24].

To our knowledge, there is no previous study comparing the electrothermal bipolar vessel sealing system for nodal dissection in patients with melanoma. In the present study, the use of the LSJ instrument for nodal dissection in cutaneous melanoma was associated with reduced postoperative lymph output and reduced duration of suction drainage. The drainage volume at postoperative day 10 was reduced by one-third compared with conventional electrocautery data. Even more than the reduction in time to drainage removal, this shows the ability of electrothermal bipolar vessel sealing to reduce postoperative lymph production (Figure 1). These findings suggest that the LSJ can be safely used in nodal dissection as an alternative to traditional methods in patients with cutaneous melanoma. However, this study presents some limitations about resctricted follow-up time and number of patients observed. A larger sample size and a long term observation are needed to confirm these data.

\section{Conclusions}

It's first report of axillary and ilio-inguinal lymph node dissection in patients with metastatic cutaneous melanoma using LSJ. The routine use of the devise was significantly associated with reduced postoperative lymph production and days of suction drain than patients treated surgically with conventional electrocautery.

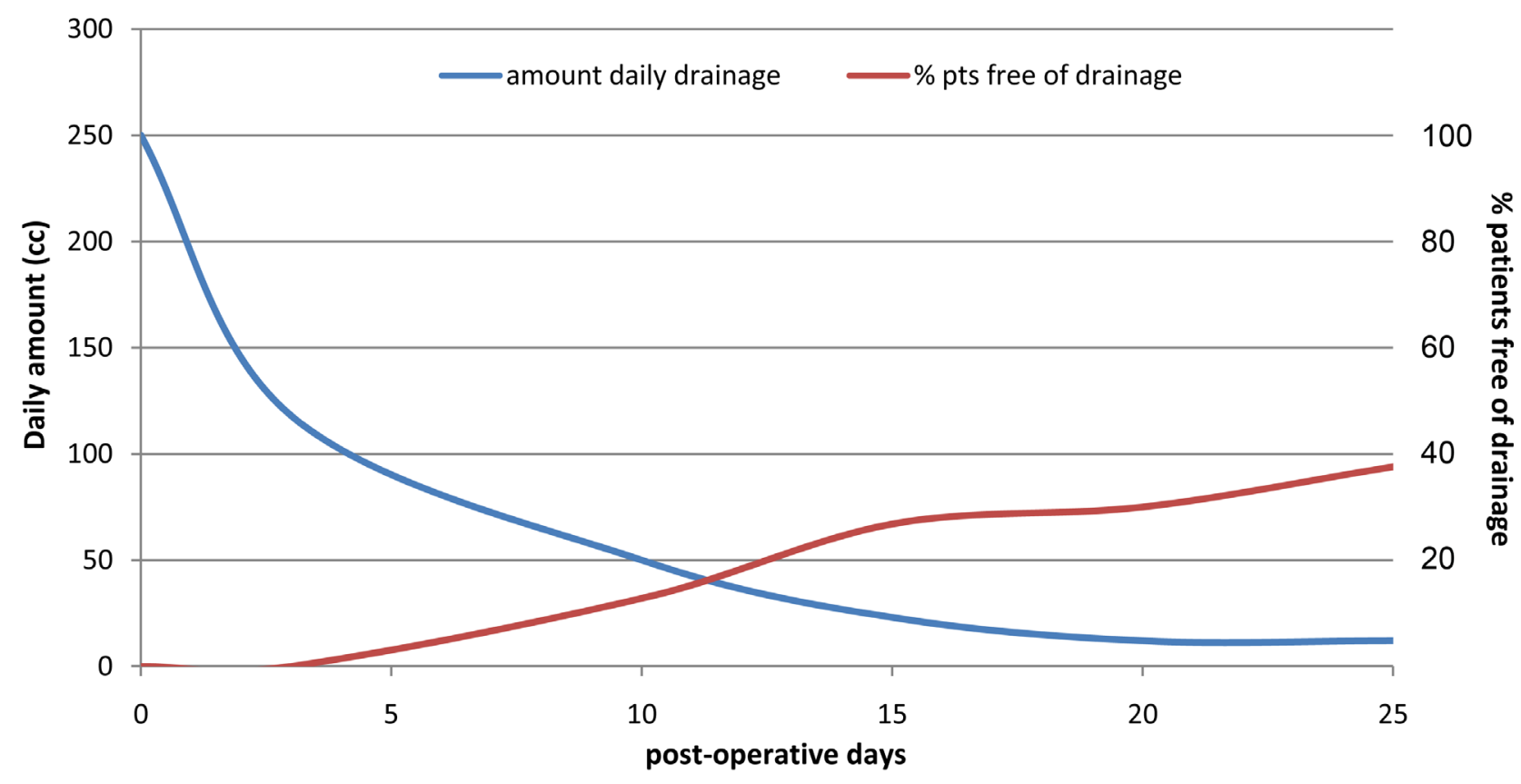

Figure 1. Daily amount of drainage and percentage of patients free of drainage according post-operative days. 
Moreover, further studies are necessary to confirm the feasibility of the electrothermal bipolar vessel sealing system in reducing postoperative complications of node dissection in melanoma patients.

\section{Conflicts of Interest}

The authors declare no conflicts of interest regarding the publication of this paper.

\section{References}

[1] Tonouchi, H., Ohmori, Y., Kobayashi, M., Konishi, N., Tanaka, K., Mohri, Y., et al. (2004) Operative Morbidity Associated with Groin Dissections. Surgery Today, 34, 413-418. https://doi.org/10.1007/s00595-003-2738-5

[2] Mortenson, M.M., Xing, Y., Weaver, S., Lee, J.E., Gershenwald, J.E., Lucci, A., et al. (2008) Fibrin Sealant Does Not Decrease Seroma Output or Time to Drain Removal Following Inguino-Femoral Node Dissection in Melanoma Patients: A Randomized Controlled Trial. World Journal of Surgical Oncology, 6, 63-70. https://doi.org/10.1186/1477-7819-6-63

[3] Bland, K.I., Klamer, T.W., Polk Jr., H.C. and Knutson, C.O. (1981) Isolated Regional Lymph Node Dissection: Morbidity, Mortality and Economic Consideration. Annals of Surgery, 193, 372-376. https://doi.org/10.1097/00000658-198103000-00022

[4] Urist, N.M., Maddox, W.A., Kennedy, J.E. and Balch, C.M. (1983) Patients Risk Factor and Surgical Morbidity after Regional Lymphadenectomy in 204 Melanoma Patients. Cancer, 51, 2152-2156. https://doi.org/10.1002/1097-0142(19830601)51:11<2152::AID-CNCR2820511134> 3.0.CO;2-7

[5] Kuroi, K., Shimozuma, K., Taguchi, T., Imai, H., Yamashiro, H., Ohsumi, S., et al. (2006) Effect of Mechanical Closure of Dead Space on Seroma Formation after Breast Surgery. Breast Cancer, 13, 260-265. https://doi.org/10.2325/jbcs.13.260

[6] Schultz, I., Barholm, M. and Grondal, S. (1997) Delayed Shoulder Exercises in Reducing Seroma Frequency after Modified Radical Mastectomy: A Prospective Randomized Study. Annals of Surgical Oncology, 4, 293-297.

https://doi.org/10.1007/BF02303577

[7] Shamley, D.R., Barker, K., Simonite, V. and Beardshaw, A. (2005) Delayed versus Immediate Exercises Following Surgery for Breast Cancer: A Systematic Review. Breast Cancer Research and Treatment, 90, 263-271. https://doi.org/10.1007/s10549-004-4727-9

[8] Rice, D.C., Morris, S.M., Sarr, M.G., Farnell, M.B., van Heerden, J.A., Grant, C.S., et al. (2000) Intraoperative Topical Tetracycline Sclerotherapy Following Mastectomy: A Prospective Randomized Trial. Journal of Surgical Oncology, 73, 224-227. https://doi.org/10.1002/(SICI)1096-9098(200004)73:4<224::AID-JSO7>3.0.CO;2-0

[9] Galatius, H., Okholm, M. and Hoffmann, J. (2003) Mastectomy Using Ultrasonic Dissection: Effect on Seroma Formation. Breast, 12, 338-341. https://doi.org/10.1016/S0960-9776(03)00110-3

[10] Burak Jr., W.E., Goodman, P.S., Young, D.C. and Farrar, W.B. (1997) Seroma Formation Following Axillary Dissection for Breast Cancer: Risk Factors and Lack of Influence of Bovine Thrombin. Journal of Surgical Oncology, 64, 27-31. https://doi.org/10.1002/(SICI)1096-9098(199701)64:1<27::AID-JSO6>3.0.CO;2-R

[11] Moore, M., Burak, W., Nelson, E., Kearney, T., Simmons, R., Mayers, L., et al. 
(2001) Fibrin Sealant Reduces the Duration and Amount of Fluid Drainage after Axillary Dissection. A Randomized Prospective Clinical Trial. Journal of the American College of Surgeons, 192, 591-599. https://doi.org/10.1016/S1072-7515(01)00827-4

[12] Mustonen, P.K., Harma, M.A. and Eskelinen, M.J. (2004) The Effect of Fibrin Sealant Combined with Fibrinolysis Inhibitor on Reducing the Amount of Lymphatic Leakage. Scandinavian Journal of Surgery, 93, 209-212. https://doi.org/10.1177/145749690409300307

[13] Mao, X.C., Chen, C. and Wang, K.J. (2018) Efficacy and Safety of LigaSure ${ }^{\mathrm{Tm}}$ Small Jaw Instrument in Thyroidectomy: A 1-Year Prospective Observational Study. European Archives of Oto-Rhino-Laryngology, 275, 1257-1263. https://doi.org/10.1007/s00405-018-4912-9

[14] Di Monta, G., Caracò, C., Crispo, A., Marone, U. and Mozzillo, N. (2012) Collagen Sealant Patch to Reduce Lymphatic Drainage after Lymph Node Dissection. World Journal of Surgical Oncology, 10, 275. https://doi.org/10.1186/1477-7819-10-275

[15] Mc Caul, J.A., Aslaam, A., Spooner, R.J., Louden, I., Cavanagh, T. and Purushotham, A.D. (2000) Aetiology of Seroma Formation in Patients Undergoing Surgery for Breast Cancer. Breast, 9, 144-148. https://doi.org/10.1054/brst.1999.0126

[16] Agrawal, A., Ayantunde, A.A. and Cheung, K.L. (2006) Concepts of Seroma Formation and Prevention in Breast Cancer Surgery. ANZ Journal of Surgery, 76, 1088-1095. https://doi.org/10.1111/j.1445-2197.2006.03949.x

[17] Neuss, H., Raue, W., Koplin, G., Schwenk, W., Reetz, C. and Maal, J.W. (2009) A Prospective Randomized Trial: The Influence of Intraoperative Application of Fibrin Glue after Radical Inguinal/Iliacal Lymph Node Dissection on Postoperative Morbidity. European Journal of Surgical Oncology, 35, 884-889. https://doi.org/10.1016/j.ejso.2008.09.016

[18] Hoefer Jr., R.A., DuBois, J.J., Ostrow, L.B. and Silver, L.F. (1990) Wound Complications Following Modified Radical Mastectomy: An Analysis of Perioperative Factors. The Journal of the American Osteopathic Association, 90, 47-53.

[19] Neuss, H., Raue, W., Koplin, G., Schwenk, W., Reetz, C. and Mall, J.W. (2008) Intraoperative Application of Fibrin Sealant Does Not Reduce the Duration of Closed Suction Drainage Following Radical Axillary Lymph Node Dissection in Melanoma Patients: A Prospective Randomized Trial in 58 Patients. World Journal of Surgery, 32, 1450-1455. https://doi.org/10.1007/s00268-007-9461-0

[20] Coiro, S., Frattaroli, F.M., De Lucia, F., Manna, E., Fabi, F., Frattaroli, J.M., et al. (2015) A Comparison of the Outcome Using Ligasure ${ }^{\mathrm{max}}$ Small Jaw and Clamp-and-Tie Technique in Thyroidectomy: A Randomized Single Center Study. Langenbeck's Archives of Surgery, 400, 247-252. https://doi.org/10.1007/s00423-014-1270-y

[21] Hwang, S.O., Jung, J.H., Park, H.Y. and Kim, W.W. (2014) A Prospective, Randomized Study between the Small Jaw and the Harmonic Focus in Open Thyroidectomy. Otolaryngology_Head and Neck Surgery, 150, 943-948. https://doi.org/10.1177/0194599814527730

[22] Macario, A., Dexter, F., Sypal, J., Cosgriff, N. and Heniford, B.T. (2008) Operative Time and Other Outcomes of the Electrothermal Bipolar Vessel Sealing System (LigaSure) versus Other Methods for Surgical Hemostasis: A Meta-Analysis. Surgical Innovation, 15, 284-291. https://doi.org/10.1177/1553350608324933

[23] Cortadellas, T., Córdoba, O., Espinosa-Bravo, M., Mendoza-Santin, C., Rodriquez Fernandez, J., Esqueva, A., et al. (2011) Electrothermal Bipolar Vessel Sealing System in Axillary Dissection: A Prospective Randomized Clinical Study. International 
Journal of Surgery, 9, 636-640. https://doi.org/10.1016/j.ijsu.2011.08.002

[24] Tukenmez, M., Agcaoglu, O., Aksakal, N., Destek, S., Cabioglu, N., Barbaros, U., et al. (2014) The Use of Ligasure Vessel Sealing System in Axillary Dissection; Effect on Seroma Formation. Chirurgia, 109, 620-625. 